\title{
Nuevos métodos de la enseñanza-aprendizaje por medio de la tecnología en plataformas educativas
}

\section{New teaching-learning methods through technology in educational platforms}

Kleber Antonio Luna Altamirano. ${ }^{1}$, Erika Roxana Bautista Tapia. ${ }^{2}$, Gissela Nataly Rocano Pérez. ${ }^{3}$ \& Jorge Patricio Chunchi Zhingri. ${ }^{4}$

\begin{abstract}
The Educational Unit Luis Cordero located in CuencaEcuador today the educational commun ity is guided by a vision and mission aimed at the formation of values to provide quality educ ation and warmth, focused on the principles of good living media education today is essential for different learning processes are new technologies and teaching methods this is a pedagogi cal complement to the students undoubtedly this implies progress for education, as we must a dapt to new changes in life.The problem of the investigation lies in the lack of knowledge in $t$ he educational unit \&quot;Luis Cordero Crespo"; since some of them have virtual platforms t o deliver their tasks or to carry out their respective trainings, on the other hand, there are insti tutions that are totally unaware of it, for that reason it has taken that they leave aside their virt ual platforms without taking the due advantage of them since students do not know how to us e a suitable wood since they cannot use it to check their grades or to receive homework for th eir homes. The objective is to raise a training plan to improve the use of virtual platforms in o rder to know each item that contains the platform and its benefits as it can help the teaching a nd learning of teachers and students of the educational unit \&quot;Luis Cordero"; The metho dology is descriptive, with a quantitative approach, the method applied is the deductive, the $\mathrm{i}$ nformation will be obtained through the technique of the survey. The result with the help of $t$ he respondents have shown the lack of knowledge of the operation of the platform where thro ugh video tutorials, and with the help of a good training where they can answer all the doubts so the educational unit Luis Cordero of the city Cuenca Ecuador has the purpose that teachers and students are coupled in the student platforms through the internet and propose new meth ods of teaching and learning.
\end{abstract}

Keywords: Teaching, learning, education, technology, Training plan.

\footnotetext{
${ }^{1}$ Doctor (c) en Ciencias Sociales Mención Gerencia Universidad del Zulia República Bolivariana de Venezuela, Profesor Investigador Unidad Académica de Administración Universidad Católica de Cuenca, klunaa@ucacue.edu.ec

2 Estudiante de la carrera de Contabilidad y Auditoría en la Unidad Académica de Administración de la Universidad católica de Cuenca, erika.bautista.60@est.ucacue.edu.ec

3 Estudiante de la carrera de Contabilidad y Auditoría en la Unidad Académica de Administración de la Universidad Católica de Cuenca, gissela.rocano.91@est.ucacue.edu.ec

4 Estudiante de la carrera de Contabilidad y Auditoría en la Unidad Académica de Administración de la Universidad Católica de Cuenca, Jorge.chunchi.84 @est.ucacue.edu.ec
} 


\section{Resumen}

La Unidad Educativa Luis Cordero ubicada en Cuenca-Ecuador, hoy en día la comunidad educativa está orientada por una visión y misión encaminada a la formación en valores para brindar una educación de calidad y calidez, enfocada en los principios del buen vivir la educación en medios de comunicación hoy en día es indispensable para los diferentes procesos de aprendizaje se trata de nuevas tecnologías y métodos de enseñanza este es un complemento pedagógico de los alumnos sin duda esto implica un avance para la educación, ya que debemos adaptarnos a los nuevos cambios de la vida. El problema de la investigación radica en el desconocimiento en la unidad educativa "Luis Cordero Crespo" ya que algunas poseen unas plataformas virtuales para entregar sus tareas o realizar sus respectivas capacitaciones, por otra parte, existen instituciones que desconocen total mente de ello, por ello ha llevado que dejen a un lado sus plataformas virtuales sin sacarles el debido provecho ya que alumnos no saben utilizar de una madera adecuada ya que no pueden utilizar para revisar sus notas o recibir deberes para su hogar. El objetivo es plantear un plan de capacitación para mejorar la utilización de las plataformas virtuales con el fin que conozcan cada ítem que contiene la plataforma y sus beneficios ya que puede ayudar a la enseñanza y aprendizaje de los profesores y estudiantes de la unidad educativa "Luis Cordero "La metodología es de tipo descriptivo, con enfoque cuantitativo, el método aplicado es el deductivo, la información se obtendrá a través de la técnica de la encuesta. El resultado con la ayuda de los encuestados han de mostrado la falta de conocimiento del funcionamiento de la plataforma donde por medio de videos tutoriales, y con la ayuda de una buena capacitación donde puedan responder todas las dudas así la unidad educativa Luis Cordero de la cuidad Cuenca -Ecuador tiene el propósito que los profesores y estudiantes estén acoplados en las plataformas estudiantiles mediante el internet y proponerse nuevos métodos de enseñanza y aprendizaje.

Palabras claves: Enseñanza, Aprendizaje, Educación, Tecnología, Plan de capacitación.

\section{Introducción}

La Unidad Educativa Luis Cordero Crespo está ubicada en la ciudad de Cuenca -Ecuador sus inicios fue en el año 1900 la actual directora es Mg. Gladis Segarra, por muchos años llevo la denominación de escuela superior debido a la alta calidad de educación impartida en sus aulas. La escuela Luis Cordero Crespo cumplido su primer centenario de vida institucional el 15 de mayo del 2017 ; siendo la casa de cientos de cuencanos ,educando a decena de promociones .por sus aulas han desfilado estudiantes que son grades personalidades que se han destacado en diferentes campos ,prueba de ello el Dr. Mario Jaramillo Paredes, en la actualidad la institución cuenta con más de 1700 estudiantes en su jornada matutina y vespertina, con una oferta educativa desde el Nivel inicial hasta el Décimo grado de Educación General Básica, hoy en día la comunidad educativa de la escuela Luis Cordero Crespo está orientada por una visión y misión encaminada a la formación en valores para brindar una educación de calidad y calidez, enfocada en los principios del buen vivir.

El problema radica en el desconocimiento de parte de los estudiantes y de los profesores que no han puesto en práctica una plataforma para sus respectivas actividades donde les ayudaría de tal manera que en casa como en clases se sientan cómodos con las tareas a realizarse y de la misma manera los profesores no han recibido una metodología que los estudiantes se acoplen con el uso de la tecnología basándose en las plataformas educativas.

De todos es conocido el fracaso del sistema tradicional donde un profesor, después de explicar una materia, facilita un listado de problemas a los alumnos, despreocupándose tanto de si los saben hacer, como de si realmente los hacen. Ante este problema decidimos desarrollar una plataforma que asignaría problemas a cada alumno (Soler, Prados, Boada y Poch,2006, p.581). 
El objetivo de esta investigación, es diseñar de un plan de capacitación para mejorar la utilización y el conocimiento de las plataformas virtuales cuyo propósito es que puedan conocer cada parte del aula virtual. Como también se podría conocer sobre las dificultades que tienen los docentes y los estudiantes respecto a la utilización del mismo, ya que así la Unidad Educativa puede lograr una mejor enseñanza-aprendizaje mediante las plataformas

La capacitación se convierte así, en la herramienta esencial para el alcance de estos objetivos institucionales y en la estrategia educativa y de cambio que permitiría fortalecer las competencias del personal, generando diversos espacios de aprendizaje individual y colectivo, dirigido a mejorar y optimizar las habilidades, actitudes, aptitudes y conocimientos asociadas al buen desempeño de las funciones que se les establecen (Vergel, Parra, Martines,2013, p.206).

En lo metodológico, la investigación es de tipo descriptivo, con enfoque cuantitativo, con ayuda de encuestas de investigación, se logrará la mejor comprensión de las necesidades de los profesores y estudiantes de los problemas que embarca a la hora de utilizar una plataforma virtual, por ello se hace necesario capacitar a los miembros de esta Unidad Educativa, con el propósito de mejorar la enseñanza dentro del aula de clase.

El término diseño en el marco de una investigación cuantitativa se refiere al abordaje general que se utiliza en el proceso de investigación, es más flexible y abierto, y el curso de las acciones se rige por el campo (los participantes y la evolución de los acontecimientos), de este modo, el diseño se va ajustando a las condiciones del escenario o ambiente (Salgado,2007, p.72).

La finalidad de la Investigación-Acción es resolver problemas cotidianos e inmediatos, y mejorar prácticas concretas. Su propósito fundamental se centra en aportar información que guíe la toma de decisiones para programas, procesos y reformas estructurales (Salgado,2007, p.73).

El estudio este compuesto, en primer plano por la introducción, donde se da a conocer el problema que tiene la institución, el objetivo es diseñar un plan de capacitación para el mejoramiento del uso de la tecnología basándose en el aprendizaje y enseñanza, en segundo plano se trata de la metodología, mismo que tiene un enfoque cuantitativo con el apoyo de las encuestas dirigido a profesores y estudiantes, se obtiene información relevante para el aporte de este estudio, en cuanto a los resultados que se busca cambiar la forma de enseñar y aprender, finalmente como conclusión se podría dar una posible solución apoyándose en las capacitaciones dirigido a estudiantes y docentes, con la finalidad de adaptarse a los nuevos métodos tecnológicos ofrecidos dentro de la educación.

\section{Estado del arte}

Los métodos de enseñanza y aprendizaje mediante la tecnología están basados en la utilización de plataformas educativas virtuales donde tendrán un mejor conocimiento en las distintas áreas. Al diseñar un plan de capacitación se trata de dar solución al problema de la investigación en cuanto a la parte tecnológica dentro de la unidad educativa "Luis Cordero Crespo", por este motivo los siguientes autores aportaran con sus investigaciones en la parte teórica dando mayor fuerza la presente investigación entre ellos:

Echeverría (2000) diseñan nuevas formas de aprendizaje a través de la tecnología están transformando las sociedades ya que es la mejor forma de enseñar a estudiantes. Ledo y Cruz (2008) afirman que los medios de enseñanza son diseñados para utilizarlos en los procesos educativos y como recursos, otros tienen propósitos diferentes son adaptados por los docentes para procesos educativos, hace muchos años y la reciente tecnología educativa, sirven de apoyo para la efectividad de los trabajos en casa. Shutterstock (2017) al trabajar en equipo se le llama aprendizaje colaborativo, ya que el objetivo es que los alumnos adquieran los mejores de los 
conocimientos, donde los estudiantes entre si intercambian información, hasta que todos hayan terminado y entendido a través de ayudarse entre todos los compañeros del grupo.

Mato (2011) presenta un método audio lingual que también se puede decir audio-oral este sujeto con la investigación lingüística, en estados unidos tomaron medidas para estos nuevos métodos con un lenguaje determinado ya que tiene un procedimiento descriptivo y deductivo para mayor aprendizaje. Laborí ,Echeverría y Aguirre(2001) proponen en la educación enfocada en la informática tiene una visión global donde disponen de una simulación que disponen plantear sus propios casos del sistema del que se quiera estudiar, ya que cada estudiante construya sus conocimientos en aulas virtuales, la educación que llevan a cabo son estímulos sistemáticos con refuerzos de rapidez, es una de las aplicaciones modelos que llevan a instituciones donde quisieran aprender de las nuevas tecnologías, con ayuda de programas ,donde el ordenador también puede ayudar con información teórica ,también con la ayuda de un tutorial donde un procesador de la misma manera tiene una determinada rama de conocimientos.

Gros (2012) propone que el uso de las tecnologías mediante el internet genera la mayor obtención de información más amplia, donde se podría ver todas las complicaciones de los estudiantes que nos permite mirar el comportamiento de cada uno de los alumnos con la ayuda de la retroalimentación. Garcés (2019) propone que el maestro de clase para acoplarse tiene que dominar las nuevas técnicas de enseñanza en la tendencia de tecnología, y adaptarse en las aulas con la ayuda de las TIC, así podrá conocer la actividad que debe realizar con los estudiantes, en la educación virtual así los estudiantes seguirán aprendiendo según sus intereses de autoaprendizaje. Carrió (2007) expone, que las tecnologías basándose en la información y la comunicación del aprendizaje en un buen instrumento para un mejor aprendizaje ya que la dependencia actual es el internet ya que son medios para aprender y enseñar con rapidez y de una manera eficaz.

Barbera y Badia (2005) expone que un aula virtual se puede utilizar en una misma aula de clase ya que funcionada de una manera de autoevaluación en la educación superior, aporta en una cantidad de necesidades ya que el profesor y el alumno estén conectados en el mismo habito ya que esto ofrece flexibilización. Campus (2016) afirma que en los últimos años ha existido varios cambios tecnológicos lo cual ayuda a la mejora de actividades a los sectores que realizan trabajos en diferentes áreas como así también ha sido un gran cambio en la sociedad. Ramírez Castro Paniagua A y Melchor A (2018) proponen nuevos métodos de enseñanza para la medicina aprovechando la tecnología que en la actualidad existe para el aprendizaje de estudiantes y poder superarse más a nivel educativo ya que esto le ayudara a la mejora de su aprendizaje.

El siguiente articulo está compuesto como una breve introducción donde nos muestra la historia de la Unidad Educativa Luis Cordero Crespo ,con un explicación breve sobre la problemática en las plataformas virtuales, seguido de un estado del arte donde varios autores dan relevancia a la investigación ,modelo de un plan de capacitación que de la misma manera podría ayudar como una guía para ayudar a la implementación de la plataforma, finalmente muestra resultados de los encuestados que busca la manera de implementar el nuevo método de enseñanza y aprendizaje

\section{Metodología}

La presente investigación es de tipo descriptivo con enfoque cuantitativo, por lo que se aplica un método deductivo para un diseño de un plan de capacitación, la información es suministrada a través de una encuesta dirigida a 101 personas, entre ellas profesores y estudiantes de la 
Unidad Educativa Luis Cordero Crespo. A continuación, se detalla los cargos de las personas a quienes estarán dirigida la encuesta:

Tabla1. Miembros de la Unidad educativa "Luis Cordero Crespo"

\begin{tabular}{ccc}
\hline Personal total & Número & \\
\hline Docentes & 20 & \\
Estudiantes & 81 & \\
Total & & 101 \\
\hline
\end{tabular}

Fuente: Elaboración propia

\section{Resultados}

Como se puede ver en la tabla 1, un porcentaje de miembros de la Unidad Educativa Luis Cordero de la Ciudad de cuenca, se realizó una encuesta a 20 profesores y 81 estudiantes del décimo año ya que conocen el problema la mayoría de personas sobre el desconociendo de la plataforma educativa. La información más relevante de las encuestas se muestra a continuación:

Figura1.Conocimiento de las plataformas educativas

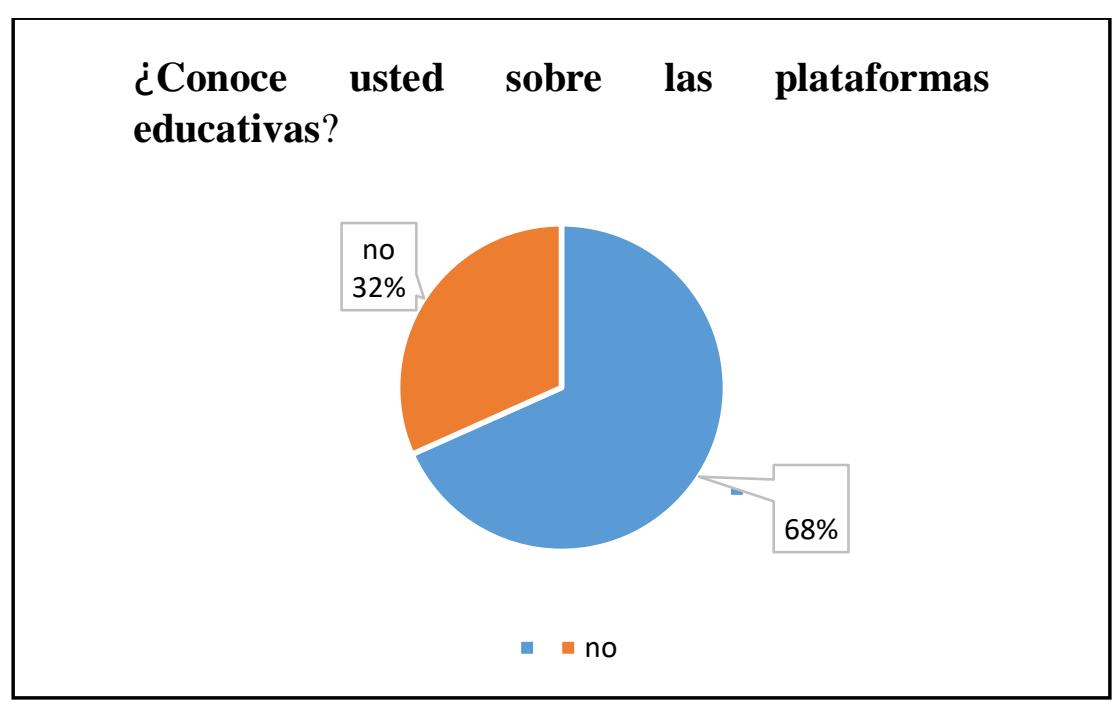

Fuente: Elaboración propia

En la suma de la respuesta se puede verificar que el $69 \%$ de los encuestados conocen sobre las plataformas educativas, pero no pueden poner en prácticas por falta de explicación de capacitación, no han recibido una mejor explicación de cómo ingresar a ese tipo de sistema, muy poca información recibida o no muy bien explicada. Por ello Ayllón (2010) Propone fomentar el aula virtual hacerle funcionar con la adecuada enseñanza mediante capacitaciones donde el $100 \%$ este al tanto de que se trata y cuáles son los propósitos que se quiere obtener 
Figura.2 aprender y enseñar mediante la plataforma educativa

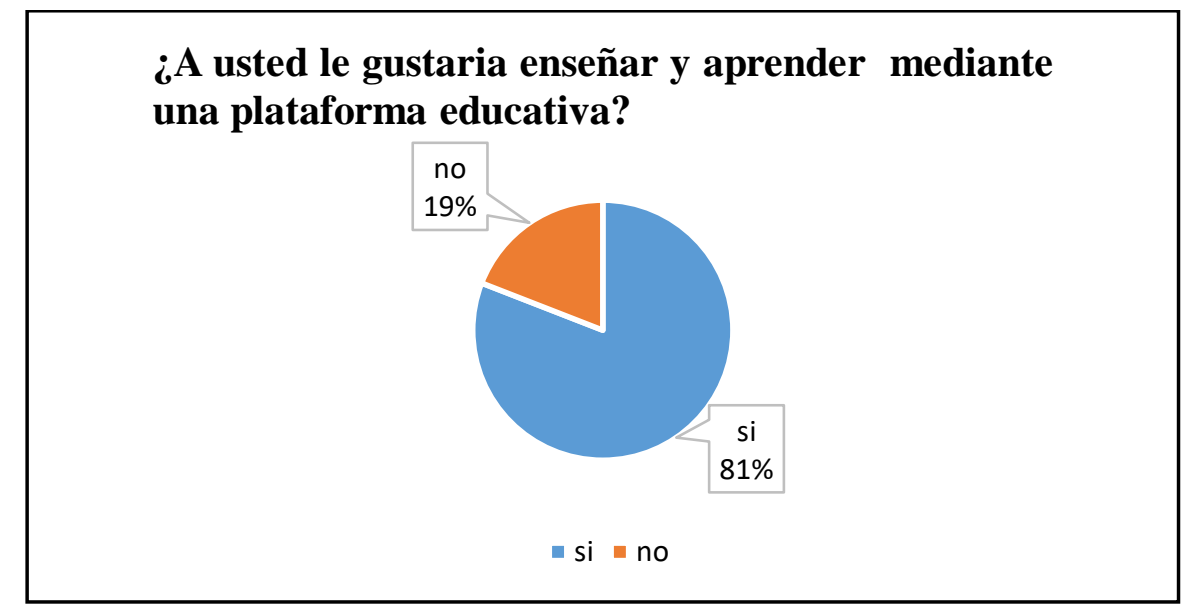

Fuente: elaboración propia

Respecto a este resultado el $77 \%$ de los encuestados respondieron que si les gustaría enseñar y aprender mediante la tecnología ya que sería una nueva fuente que les gustara conocer más a fondo saber de todas las herramientas para su beneficio a futuro. Bandia, Chumpitaz, Vargas, Suarez (2016) determinan que hay una relación positiva al tener un uso frecuente de la tecnología en las clases ya que es un reto comenzar a manipular una plataforma ya que tiene muy buenos beneficios si se sabe usar tendrá una buena formación educativa.

Figura 3. Ventajas de las plataformas educativas

\section{¿Qué ventajas traería enviar tareas por medio de las plataformas educativas?}

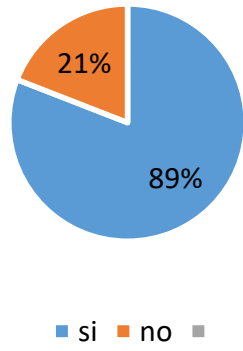

Fuente: Elaboración propia

El resultado de las siguientes respuestas el $61 \%$ de los encuestados optaron que la ventaja más alta de las plataformas educativas seria la facilitación de la entrega de deberes a los profesores ya que tanto estudiantes como profesores son indicaban que sería la mejor clave para la responsabilidad estar atentos a la hora de entrega de la tarea. Diaz (2009) sostiene que las ventajas que ha llevado las plataformas estudiantiles son de gran ayuda para el aprendizaje y enseñanza ya que es una gran iniciativa implementar una plataforma ya que ayudara tanto a los profesores como estudiantes en las distintas ramas que se pondrá en prácticas para buenos resultados estudiantiles. 
Figura 4. Conocimiento de las plataformas educativas

¿Cómo le gustaría a usted conocer más sobre las plataformas virtuales educativas?

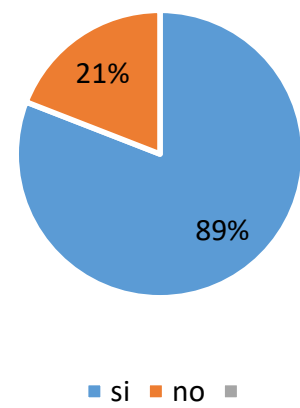

Fuente: Elaboración propia

Se puede visualizar que el $43 \%$ de los encuestados les gustaría conocer más las plataformas virtuales mediante videos tutoriales con mayor factibilidad que cada vez que quieran entrar o revisar como usar la plataforma poder repetir los videos y seguir paso a paso en segundo lugar tenemos con el $42 \%$ las capacitaciones donde se podría lograr una mejor comprensión paso a paso de igual manera con documentación física. Ayllon (2019) propone capacitaciones con ayuda del tic en forma verbal y física, conociendo los problemas, las necesidades de los estudiante y profesores, vídeos explicativos donde incentiven el dónde de aprender a conocer la plataforma virtual.

Figura 5. Implementación de la plataforma estudiantil

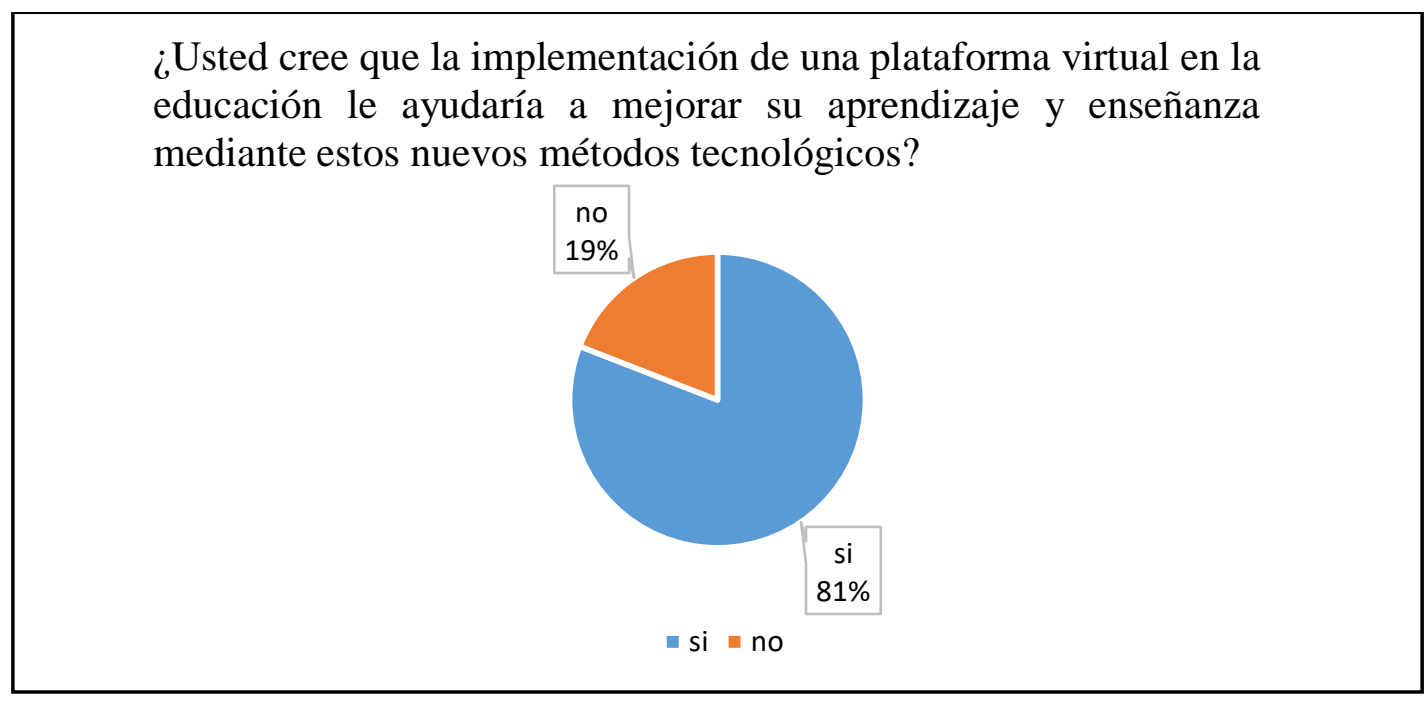

Fuente: Elaboración Propia

Se pueden evidenciar que el $89 \%$ de los encuestados están totalmente de acuerdo la implementación, el conocimiento y para poner en práctica la plataforma virtual ya que le ayudara de mucho en la forma académica tanto en profesores y estudiantes. Espinoza (2010) proyecta a un plan de capacitación antes de usar la plataforma implementada o puesta en práctica donde conozcan cada paso que deben seguir, analizar todos los procesos, ventajas y desventajas posibles. 
Partiendo de la información obtenida en las encuestas, y observando la necesidad de elaborar un plan de capacitación en el manejo de plataformas virtuales educativas, se presenta a continuación este plan con la finalidad de mejorar el proceso de enseñanza dentro del aula de clase en la Unidad Educativa Luis Cordero Crespo.

Tabla 2. Plan de capacitación en plataformas virtuales

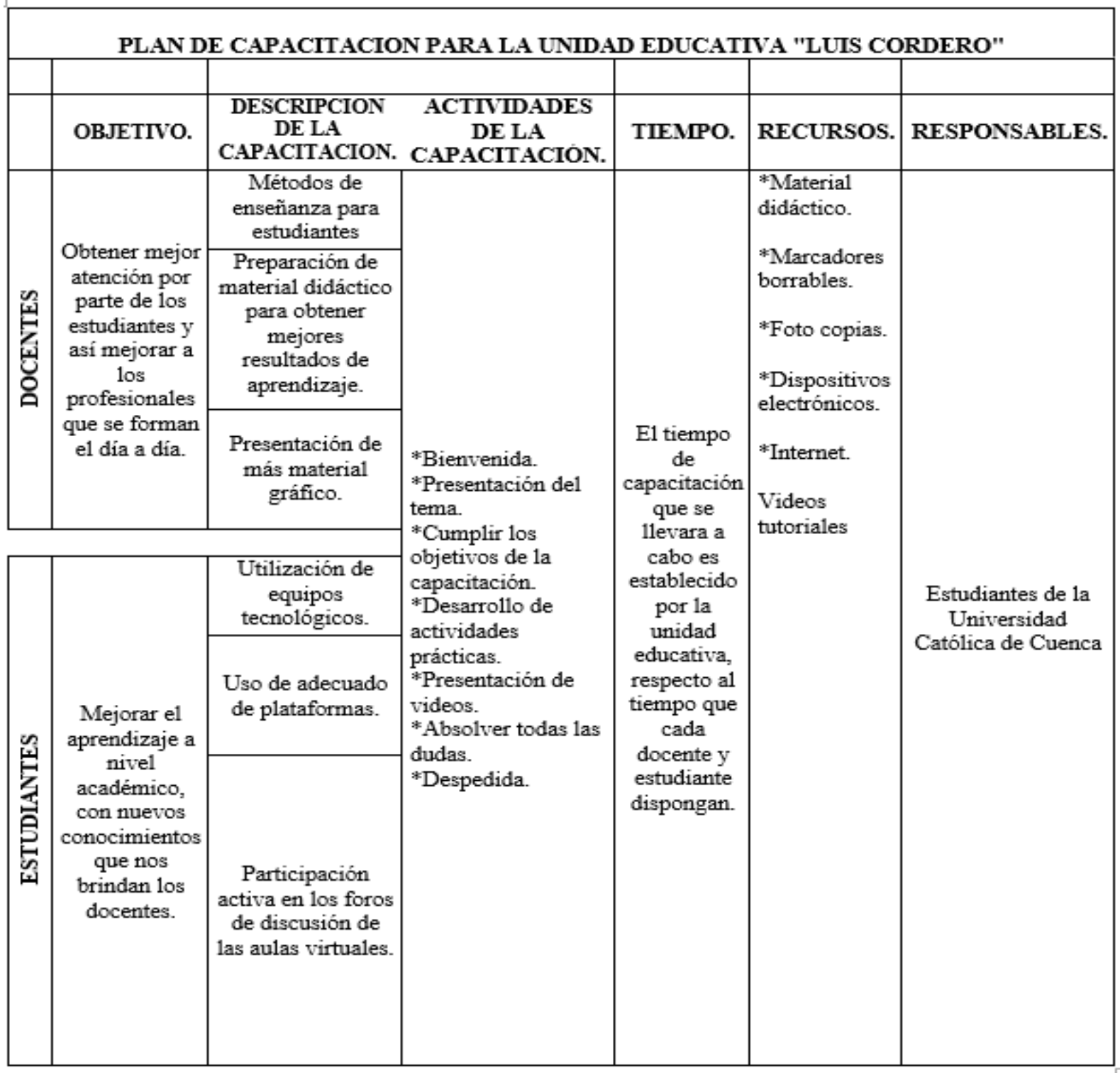

Fuente: Elaboración propia

En la tabla número 2 , se observa el diseño del plan de capacitación sobre las plataformas virtuales en la Unidad Educativa Luis Cordero tanto para estudiantes y docentes, la finalidad es utilizar de manera eficiente la plataforma virtual educativa, poniendo en práctica de manera permanente, con ello se trata de mejorar el nivel académico, y utilizar nuevos medios de enseñanza-aprendizaje, este plan contempla medios didácticos como videos tutoriales donde será efectivo para el buen aprendizaje, se realizará una discusión o foros sobre el conocimiento. 
La capacitación ira siempre alineado a los objetivos institucionales, con ello se logrará una mejor enseñanza dentro de esta institución educativa.

Es necesario explicar las ventajas y desventajas de la utilización de estas plataformas virtuales, ya que las mismas siempre estarán alineadas a ampliar el conocimiento.

Tabla 3. Ventajas y Desventajas de la utilización de plataformas virtuales

\begin{tabular}{|l|l|}
\hline \multicolumn{1}{|c|}{ VENTAJAS } & \multicolumn{1}{|c|}{ DESVENTAJAS } \\
\hline $\begin{array}{l}\text { Ampliar la comunicación entre docente } \\
\text { y alumno, de manera del transcurso } \\
\text { como emplee herramientas en la } \\
\text { plataforma. }\end{array}$ & $\begin{array}{l}\text { Que no todos lo estudiantes tienen la } \\
\text { facilidad de adaptarse a un nuevo } \\
\text { medio tecnológico. }\end{array}$ \\
\hline $\begin{array}{l}\text { Mayor facilidad en el acceso de la } \\
\text { búsqueda de la informacion } \\
\text { correspondiente que el docente } \\
\text { implemente. }\end{array}$ & $\begin{array}{l}\text { Falta de una buena capacitación para el } \\
\text { manejo de la plataforma. }\end{array}$ \\
\hline $\begin{array}{l}\text { Facilidad de entrega de tareas donde } \\
\text { toda la información queda respaldada. }\end{array}$ & $\begin{array}{l}\text { Problemas técnicos y costos elevados } \\
\text { del mantenimiento. }\end{array}$ \\
\hline $\begin{array}{l}\text { Estaría al tanto de los trabajos enviados } \\
\text { por el docente y sus compañeros. }\end{array}$ & $\begin{array}{l}\text { Que la plataforma no funcione de una } \\
\text { manera correcta y no pueda realizar las } \\
\text { actividades propuestas por el docente. }\end{array}$ \\
\hline $\begin{array}{l}\text { El medio tecnológico se podría } \\
\text { considerar para los estudiantes como } \\
\text { una materia interesante. }\end{array}$ & $\begin{array}{l}\text { Falta de socialización entre } \\
\text { compañeros provocando aislamiento. }\end{array}$ \\
\hline $\begin{array}{l}\text { Facilita la participación individual y } \\
\text { colectiva. }\end{array}$ & $\begin{array}{l}\text { Falta de concentración a la hora de } \\
\text { realizar las tareas, desviación de otras } \\
\text { páginas web }\end{array}$ \\
\hline $\begin{array}{l}\text { Se beneficia a personas con su corto } \\
\text { tiempo ya que pueden realizar sus } \\
\text { actividades en cualquier lugar. }\end{array}$ & $\begin{array}{l}\text { Mediante el autoestudio exige al } \\
\text { estudiante un mayor esfuerzo, } \\
\text { disciplina en la formación }\end{array}$ \\
\hline
\end{tabular}

Fuente: Elaboración Propia

\section{Conclusiones}

El diseñar un plan de capacitación para La Unidad Educativa Luis Cordero Crespo de la cuidad Cuenca -Ecuador, es tartar de corregir el inconveniente que viene atravesando esta institución educativa, el desconocimiento de la operatividad de las plataformas virtuales educativas, conlleva a docentes y estudiantes a la necesidad de capacitarse en el tema referido. La propuesta presentada en este estudio, se orienta a instruir de una manera apropiada en la operatividad y eficacia de estas herramientas educativas, con ello la comunidad educativa entrará en un proceso de mejoramiento de la enseñanza-aprendizaje, con el propósito de tratar de llegar a la calidad pedagógica.

La presente investigación, enmarca un aporte al conocimiento de los docentes y estudiantes, sobre el mundo actual de la enseñanza educativa, la tecnología avanza y crea una sociedad 
diferente, por ello se debe profesar en todo el personal de esta institución educativa una cultura de capacitación, ya que la ciencia siempre estará superando barreras puestas por la sociedad.

Entregar a los directivos de esta Unidad Educativa este plan de capacitación sobre la utilización de plataformas virtuales educativas, impactará en el mejoramiento del proceso de enseñanzaaprendizaje y transmitirá a padres de familia y comunidad en general, a través de una mejor educación dentro del aula de clase, conllevando en un corto plazo a obtener una educación de calidad, con ello lograr cumplir con los ideales institucionales.

\section{Referencias Bibliográficas}

Abarca, Y. (2019). Procesos de capacitación ayudados por TIC en el ámbito laboral. Revistas de lenguas modernas, (25). https://revistas.ucr.ac.cr/index.php/rlm/article/view/27710/27927

Ayllon, J. (2010). El uso de La plataforma de enseñanza virtual para impartir asignaturas jurídicas. Revista jurídica de investigación e innovación educativa, (1), pg.49-60. Recuperado de http://www.eumed.net/rev/rejie/01/pdf/49-60_jmadg.pdf

Badia, A., Chumpitaz. L., Vargas, J. y Suárez, G. (2016). La percepción de la utilidad de la tecnología conforma su uso para enseñar y aprender. Revista Electrónica de Investigación Educativa, 18(3), 95-105. Recuperado de http://redie.uabc.mx/redie/article/view/810

Carrió, M. (2007). Ventajas del uso de la tecnología en el aprendizaje colaborativo, 4. (10). http://campus.usal.es/ ofeees/NUEVAS_METODOLOGIAS/COLABORATIVO/164 0Carrio[1].pdf

Campus, N. (2016). Metodología de enseñanza y para el aprendizaje, pg.13(1), Recuperado de https://www.nebrija.com/nebrija-global-campus/pdf/metodologia-ensenanzaaprendizaje.pdf

Castro, R., y Melchor, A (2018) Impacto de un modelo pedagógico constructivista apoyado con TIC para desarrollar competencias en medicina, pg.1(2). http://www.scielo.org.mx/scielo.php?script=sci_arttext\&pid=S2007$50572018000400035 \&$ lang=es

Contreras, A. (2019). Ambientes Virtuales de Aprendizaje. Dificultades de uso en los estudiantes de cuarto grado de primaria, 215-240. Recuperado de http://www.scielo.org.co/pdf/prsp/n27/2389-993X-prsp-27-00215.pdf

Díaz, S. (2009). Plataformas educativas en un entorno para profesores y alumnos. Revista digital para profesionales de la enseñanza, (2). Recuperado de https://www.feandalucia.ccoo.es/docu/p5sd4921.pdf

Echeverria, J. (2000). Educación y tecnologías telemáticas. Revista Ibero Americana de Educacion.http://www.eweb.unex.es/eweb/didactica/Tecnologia_Educativa/PDF/Eche verr\%EDa.PDF

Espinoza, J. (2010). TEC Digital: una Iniciativa de Implementación de e-learning en Costa Rica. Recuperado de https://www.researchgate.net/profile/Julia_EspinozaGuzman/publication/258517103_TEC_Digital_una_Iniciativa_de_Implementacion_d e_e-learning_en_Costa_Rica/links/54d8bb0c0cf24647581b4f09.pdf 
LaborÍ, B., Echeverría, J., y Aguirre, I. (2001). Estrategias Educativas para el uso de las nuevas tecnologías de la información y comunicación. Recuperado de https://rieoei.org/RIE/article/view/3008/3911

Ledo, M., y Cruzll, C. (2008). Tecnología educativa, medios y recursos de enseñanzaaprendizaje. Educación Media Superior.22(4). http://scielo.sld.cu/pdf/ems/v22n4/ems10408.pdf

Mato, N.(2011).Principales métodos de enseñanza de lenguas extranjeras en Alemania,(6).Recuperado de file:///C:/Users/Renato\%20Barrera/Downloads/Dialnet PrincipalesMetodosDeEnsenanzaDeLenguasExtranjerasE-4779301.pdf

Ramírez, C., y Melchor, A. (2018). Impacto de un modelo pedagógico constructivista apoyado con TIC para desarrollar competencias en medicina, pg.1(2) Recuperado de http://www.scielo.org.mx/scielo.php?script=sci_arttext\&pid=S2007$50572018000400035 \&$ lang=es

Salgado, A. (2007). investigación cualitativa diseños. evaluación del rigor metodológico y retos. 71-78. http://www.scielo.org.pe/pdf/liber/v13n13/a09v13n13.pdf

Soler, J., Prados, F., Boada, I., y Poch, J. (2006). Utilización de una plataforma de e-learning en la docencia de bases de datos.

file://C:/Users/BIBLIOTECA/Downloads/Utilizacion_de_una_plataforma_de_elearning_en_la_.pdf

Vergel, M., Parra, H., y Martínez, J. (2013). Metodología para elaborar planes de capacitación en instituciones de Educación superior Logos ciencia y tecnología. 5(1), 205.Recuperado de http://www.redalyc.org/articulo.oa? $\mathrm{id}=517751547019$

\section{【 Ciencia}




\section{Para citar el artículo indexado}

Luna Altamirano, K. A., Bautista Tapia, E. R., Rocano Pérez, G. N., \& Chunchi Zhingri, J. P. (2020). Nuevos métodos de la enseñanza-aprendizaje por medio de la tecnología en $\begin{array}{lllll}\text { plataformas } & \text { educativas. } & \text { Explorador } & \text { Digital, }\end{array}$ https://doi.org/10.33262/exploradordigital.v4i1.1072

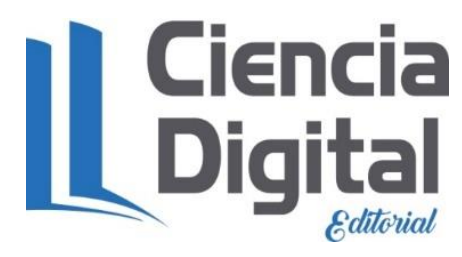

El artículo que se publica es de exclusiva responsabilidad de los autores y no necesariamente reflejan el pensamiento de la Revista Explorador Digital.

El articulo queda en propiedad de la revista y, por tanto, su publicación parcial y/o total en otro medio tiene que ser autorizado por el director o editor de la Revista Explorador Digital.

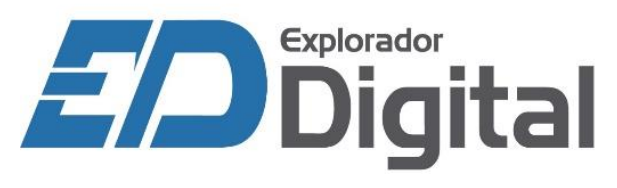

